\author{
BANACH CENTER PUBLICATIONS, VOLUME 79 \\ INSTITUTE OF MATHEMATICS \\ POLISH ACADEMY OF SCIENCES \\ WARSZAWA 2008
}

\title{
APPLICATIONS OF NONNEGATIVE OPERATORS TO A CLASS OF OPTIMIZATION PROBLEMS
}

\author{
K. C. SIVAKUMAR \\ Department of Mathematics, Indian Institute of Technology Madras \\ Chennai 600 036, India \\ E-mail: kcskumar@iitm.ac.in
}

\begin{abstract}
Let $X$ be a partially ordered real Banach space, $a, b \in X$ with $a \leq b$. Let $\phi$ be a bounded linear functional on $X$. We call $X$ a Ben-Israel-Charnes space (or a $B-C$ space) if the linear program defined by Maximize $\phi(x)$ subject to $a \leq x \leq b$ has an optimal solution for any $\phi$, $a$ and $b$. Such problems arise naturally in solving a class of problems known as Interval Linear Programs. $B-C$ spaces were introduced in the author's doctoral thesis and were subsequently studied in [8] and [9]. In this article, we review these results, study their implications to certain positive operators over partially ordered Banach spaces and obtain some new ones.
\end{abstract}

1. Introduction. Let $X$ and $Y$ be real Banach spaces with $Y$ partially ordered (Definition 2.1 below), $A: X \rightarrow Y$ be a linear map and $a, b \in Y$ with $a \leq b$. Let $\phi$ be a linear functional on $X$. A class of linear programs studied rather extensively known as interval linear programs (ILP) denoted by $\operatorname{ILP}(a, b, \phi, A)$ are problems of the form:

$$
\begin{gathered}
\text { Maximize } \phi(x) \\
\text { subject to } a \leq A x \leq b .
\end{gathered}
$$

Ben-Israel and Charnes were the first to investigate ILP's [1]. They considered the case $X=\mathbb{R}^{n}$ and $Y=\mathbb{R}^{m}$ where they assumed that the matrix $A$ is of full row-rank. Explicit optimal solutions for such problems were given in terms of generalized inverses of $A$. Kulkarni and Sivakumar [3, 4, 5] investigated interval linear programs in the infinite dimensional setting and showed how some of the results in the finite dimensional case can be extended, [8] and [9].

The study of ILP over infinite dimensional spaces naturally leads to the abstract notion of a class of Banach spaces with a certain optimization property. Such spaces were called Ben-Isreal-Charnes spaces (or $B-C$ spaces, for short), ([7]) in recognition of the

2000 Mathematics Subject Classification: Primary 90C48.

Key words and phrases: partially ordered Banach spaces, Ben-Israel-Charnes spaces.

The paper is in final form and no version of it will be published elsewhere. 
work of these authors on finite interval linear programs. Specifically, a partially ordered real Banach space $X$ is called a $B$-C space if $\operatorname{ILP}(a, b, \psi, I)$ has an optimal solution for all $a, b \in X$ with $a \leq b$ and for all bounded linear functionals $\psi$ on $X$.

A systematic study of $B-C$ spaces was carried out in [8] and [9], where new $B-C$ spaces were identifed from old ones. These were achieved by applying nonnegativity of certain operators concerned. In this article our objective is to first provide an exposition of these results. Turning around these statements lead to questions of nonnegativity of certain operators over partially ordered Banach spaces, perhaps hitherto unheard of. The second aim of this article is to pose these questions and also to consider answers to some of them. These lead to new points of view into classical results in the literature. New results in this article are given in Theorem 2.15 and Theorem 2.16.

2. Ben-Israel-Charnes spaces. In this section first we develop the terminology and review the notions of cones. We then review the existing results in the literature. We then consider questions arising out of these results. Two specific questions are answered in the affirmative and are presented in Theorem 2.15 and Theorem 2.16.

Definition 2.1. Let $X$ be a real vector space. Then $X$ is called a partially ordered vector space if $X$ has a partial order $\leq$ defined on it satisfying the following: For $x, y \in X$ with $x \leq y$, we have $x+u \leq y+u$ for all $u \in X$ and $\alpha x \leq \alpha y$ for all $\alpha \geq 0$.

Definition 2.2. Let $X$ be a partially ordered real vector space. Then the subset $\mathcal{C}:=$ $\{x \in X: x \geq \mathbf{0}\}$ is called the positive cone of $X$.

Note that $\mathcal{C}$ is a pointed cone i.e., $\mathcal{C} \cap-\mathcal{C}=\{0\}$.

Definition 2.3. A Banach space which is partially ordered is said to be a partially ordered Banach space. It is said to be a partially ordered Hilbert space, if in addition, it is a Hilbert space.

EXAMPLE 2.4. $\mathbb{R}^{n}$ the Euclidean space is a partially ordered real Banach space with $\mathbb{R}_{+}^{n}:=\left\{x=\left(x_{1}, x_{2}, \cdots, x_{n}\right) \in \mathbb{R}^{n}: x_{i} \geq 0 \forall i=1,2, \cdots, n\right\}$ as a pointed positive cone.

EXAMPLE 2.5. Let $\mu$ be a $\sigma$-finite positive measure on a $\sigma$-algebra in a nonempty set $Y$ and for $1 \leq p<\infty$, let $X=L^{p}(Y, \mu)$ denote the space of (equivalent classes of) measurable p-integrable functions on $Y$. Then $X$ is a partially ordered Banach space with the pointed positive cone $\mathcal{C}:=\{f \in X: f \geq 0$ a.e. $(\mu)\}$. In particular $l^{p}, 1 \leq p<\infty$ is a partially ordered Banach space with the pointed positive cone $\mathcal{P}:=\left\{x \in l^{p}: x_{i} \geq 0 \forall i\right\}$.

Definition 2.6. Let $X_{1}$ be a real Banach space and $X_{2}$ be a partially ordered real Banach space. Let $A: X_{1} \rightarrow X_{2}$ be linear, $\phi$ be a bounded linear functional on $X_{1}$ and $a, b \in X_{2}$ with $a \leq b$. Consider the problem denoted by $\operatorname{ILP}(a, b, \phi, A)$ :

$$
\begin{gathered}
\text { Maximize } \phi(x) \\
\text { subject to } a \leq A x \leq b
\end{gathered}
$$

A vector $x^{*} \in X_{1}$ is said to be feasible for the problem $\operatorname{ILP}(a, b, \phi, A)$ if $a \leq A x^{*} \leq b$. The problem $\operatorname{ILP}(a, b, \phi, A)$ is said to be feasible if there exists a feasible vector for it. A feasible vector $x^{*}$ is said to be optimal if $\phi\left(x^{*}\right) \geq \phi(x)$ for every feasible vector $x$. The problem $\operatorname{ILP}(a, b, \phi, A)$ is said to be bounded if $\sup \{\phi(x): a \leq A x \leq b\}<\infty$. 
Definition 2.7. Let $X$ be a partially ordered real Banach space. Let $I$ denote the identity map on $X$. We say that $X$ is a Ben-Israel-Charnes space or a $B-C$ space for short if $\operatorname{ILP}(a, b, \psi, I)$ has an optimal solution for all $a, b \in X$ with $a \leq b$ and for all bounded linear functionals $\psi$ on $X$.

The next result is a consequence of the fact that in a Banach lattice with order continuous norm, intervals of the form $[a, b]:=\{x \in X: a \leq x \leq b\}$ are weakly compact. For the notions of a Banach lattice and order complete norms, we refer to the book by Schaefer, [6].

THEOREM 2.8. Let $X$ be a Banach lattice with order continuous norm. Then $X$ is a B-C space.

The next result collects a class of Hilbert spaces that are $B-C$ spaces.

THEOREM 2.9 (Lemma 4, [3]). Let $H$ be a partially ordered real Hilbert space with $\mathcal{P}$ as the positive cone such that there exists an orthonormal basis $\left\{u^{\alpha}: \alpha \in J\right\}, J$ an index set, of $H$ with $u^{\alpha} \in \mathcal{P} \forall \alpha \in J$. Then $H$ is a $B-C$ space.

The next Theorem is a trivial consequence of Theorem 2.9.

THEOREM 2.10. Let $H$ be a partially ordered real Hilbert space with $\mathcal{P}$ as the positive cone such that there exists an orthonormal basis $\left\{u^{\alpha}: \alpha \in J\right\}, J$ an index set, of $H$ with either $u^{\alpha} \in \mathcal{P} \forall \alpha \in J$ or $u^{\alpha} \in-\mathcal{P} \forall \alpha \in J$. Then $H$ is a B-C space.

EXAMPLE 2.11. Consider the vector space $H=S \mathbb{R}^{n \times n}$ of real $n \times n$ symmetric matrices with the cone $P S D$ of positive semi-definite matrices. Identifying $S \mathbb{R}^{n \times n}$ with $\mathbb{R}^{n \times(n+1) / 2}$ it follows that $S \mathbb{R}^{n \times n}$ is a Banach lattice with order continuous norm. Hence by Theorem 2.8 , it follows that any $I L P$ posed over $S \mathbb{R}^{n \times n}$ has an optimal solution. Thus $S \mathbb{R}^{n \times n}$ is a $B-C$ space.

REMARK 2.12. It has been shown (Theorem 3.13, [9]) that the Hilbert space $H=S \mathbb{R}^{n \times n}$ with the cone $P S D$ does not satisfy the conditions of Theorem 2.9. It is not clear if this same conclusion can be arrived at, using Theorem 2.10. This leads us to the following open question:

Problem 1. Does $\mathbb{R}^{n \times(n+1) / 2}$ have an orthonormal basis $\left\{E^{k}\right\}, k=1,2, \ldots, n \times$ $(n+1) / 2$, where $E^{k} \in P S D$ or $-E^{k} \in P S D$ ?

We turn our attention to the idea of constructing new $B-C$ spaces from old.

Let $X_{1}$ and $X_{2}$ be partially ordered vector spaces with positive cones $\mathcal{P}_{1}$ and $\mathcal{P}_{2}$, respectively. Recall that a linear map $A: X_{1} \rightarrow X_{2}$ is called nonnegative if $A \mathcal{P}_{1} \subseteq \mathcal{P}_{2}$. In the rest of the paper we will use the notation $A \geq 0$ to denote this fact. Let $A: X_{1} \rightarrow X_{2}$ be linear. A linear map $T: X_{2} \rightarrow X_{1}$ is called a left-inverse of $A$ if $T A=I$, the identity map on $X_{1}$.

We now consider the following problem:

Problem 2. Let $X_{1}$ be a $B$ - $C$ space, $X_{2}$ be a partially ordered Banach space, $A: X_{1} \rightarrow$ $X_{2}$ be bounded linear with $R(A)$ closed and $A \geq 0$. When is $R(A)$ a $B-C$ space? 
Theorem 2.13 gives a sufficient condition ensuring an affirmative answer. This appeared in [9] and a sketch of its proof is given here for the sake of completeness.

THEOREM 2.13 (Theorem 3.26, [9]). Let $X_{1}$ be a B-C space and $X_{2}$ be a partially ordered real Banach space. Let $A \in B L\left(X_{1}, X_{2}\right)$ be nonnegative and $R(A)$ be closed. Suppose that $A$ has a nonnegative left-inverse. Then $R(A)$ is a $B-C$ space.

Proof. Let $y^{1}, y^{2} \in R(A)$ with $y^{1} \leq y^{2}$ and $\phi$, be a bounded linear functional on $R(A)$. Let $T$ be a nonnegative left-inverse of $A$. Set $x^{1}=T y^{1}$ and $x^{2}=T y^{2}$. Let $\psi$ be a bounded linear extension of $\phi$ to $X_{2}$. Then $\operatorname{ILP}\left(y^{1}, y^{2}, \phi, I\right)$ can be shown to be equivalent to $\operatorname{ILP}\left(x^{1}, x^{2}, A^{*} \psi, I\right)$ which has an optimal solution, as $X_{1}$ is a $B$ - $C$ space.

We next present a new application of Theorem 2.13 (Theorem 2.15 to follow). Prior to that we first quickly review the notion of the Moore-Penrose generalized inverse of an operator $T$.

Let $T \in \mathrm{BL}\left(\mathrm{H}_{1}, \mathrm{H}_{2}\right)$ with closed range and let $T^{*}$ denote the adjoint of $T$. Then the Moore-Penrose inverse of $T$ is the unique operator $T^{\dagger}$ in $\mathrm{BL}\left(\mathrm{H}_{2}, \mathrm{H}_{1}\right)$ which satisfies the following equations (See [2], Chapter II, Sec.2):

$$
\begin{gathered}
T T^{\dagger} T=T, \\
T^{\dagger} T T^{\dagger}=T^{\dagger}, \\
\left(T T^{\dagger}\right)^{*}=T T^{\dagger}, \\
\left(T^{\dagger} T\right)^{*}=T^{\dagger} T .
\end{gathered}
$$

The following properties of $T^{\dagger}$ are well known ([2]): If $x \in R\left(T^{*}\right)$ then $x=T^{\dagger} T x$; $R\left(T^{*}\right)=R\left(T^{\dagger}\right) ; T^{\dagger} T=P_{R\left(T^{*}\right)} ; N\left(T^{*}\right)=N\left(T^{\dagger}\right) ; T T^{\dagger}=P_{R(T)}$, where $T^{*}$ is the adjoint of $T, N(T)$ denotes the null space of $T, R(T)$ denotes the range space of $T$ and $P_{L}$ the orthogonal projection of a Hilbert space onto a closed subspace $L$.

REMARK 2.14. Let Le denote the lexicographic cone in $\mathbb{R}^{m}$. It can be shown that $\left(\mathbb{R}^{m}, L e\right)$ is not a $B-C$ space. In the light of Theorem 2.13 it now follows that there cannot exist a nonnegative matrix $A$ (i.e., $A\left(\mathbb{R}_{+}^{n}\right) \subseteq L e$ ) having a nonnegative left-inverse $T$ (i.e., $\left.T(L e) \subseteq \mathbb{R}_{+}^{n}\right)$ from $\left(\mathbb{R}^{n}, \mathbb{R}_{+}^{n}\right)$ into $\left(\mathbb{R}^{m}, L e\right), n \leq m$. This leads us to the following open question:

Problem 3. Let $A \in \mathbb{R}^{m \times n}$ be such that $A\left(\mathbb{R}_{+}^{n}\right) \subseteq$ Le. When is $A^{\dagger}(L e) \subseteq \mathbb{R}_{+}^{n}$ ?

Next, we review the notion of a cone and its dual. A cone $P$ in a Hilbert space $H$ is said to be acute if $\langle x, y\rangle \geq 0$ for all $x, y \in P$. For a cone $P$ in a Hilbert space $H$, the dual cone $P^{*}$ is defined by

$$
P^{*}:=\{x \in H:\langle x, y\rangle \geq 0 \forall y \in P\}
$$

A cone $P$ is said to be self-dual if $P^{*}=P$.

THEOREM 2.15. Let $P$ be a closed positive self-dual cone in a real Hilbert space $H$ which is also a $B-C$ space. Let $A: H \rightarrow H$ be a nonnegative injective bounded linear operator with $R(A)$ closed. Suppose that $\left(A^{\dagger}\right)^{*} P$ is an acute cone. Then $R(A)$ is a $B-C$ space. 
Proof. In view of Theorem 2.13, it is sufficient to demonstrate that $A$ has a nonnegative left inverse. In fact we show that $\left(A^{*} A\right)^{-1} A^{*} \geq 0$. (Note that $\left(A^{*} A\right)^{-1}$ exists as $A$ is injective. Also $A^{\dagger}=\left(A^{*} A\right)^{-1} A^{*}$, so that $A^{\dagger}\left(A^{\dagger}\right)^{*}=\left(A^{*} A\right)^{-1}$.) So, let $x \in P$ and set $y=\left(A^{*} A\right)^{-1} A^{*} x=\left(A^{*} A\right)^{-1} z, z=A^{*} x$. Since $P$ is self-dual and $A \geq 0$ we have $A x \in P^{*}$. Therefore, $\left\langle x, A^{*} u\right\rangle=\langle A x, u\rangle \geq 0$ for any $u \in P$. Consequently, $A^{*} u \in P^{*}=P$ for any $u \in P$. Now we see that $A^{*} \geq 0$ and $z \geq 0$. Next, we must show that $y \in P$. We show that if $r \in P$, then $\langle y, r\rangle \geq 0$. Since $P$ is self-dual the result would then follow. Consider $\langle y, r\rangle=\left\langle\left(A^{*} A\right)^{-1} z, r\right\rangle=\left\langle A^{\dagger}\left(A^{\dagger}\right)^{*} z, r\right\rangle=\left\langle\left(A^{\dagger}\right)^{*} z,\left(A^{\dagger}\right)^{*} r\right\rangle=\langle u, v\rangle$, where $u=\left(A^{\dagger}\right)^{*} z$ and $v=\left(A^{\dagger}\right)^{*} r$. Since $\left(A^{\dagger}\right)^{*} P$ is acute, we have $\langle u, v\rangle \geq 0$, completing the proof.

Next, we study:

Problem 4. Can the existence of a nonnegative left-inverse of $A$ in Theorem 2.13 be replaced by $A^{\dagger} \geq 0$ ?

The answer is in the affirmative for Hilbert spaces, as we prove next.

THEOREM 2.16. Let $H_{1}$ and $H_{2}$ be real Hilbert spaces with positive cones $\mathcal{P}_{1}$ and $\mathcal{P}_{2}$, respectively. Let $A \in B L\left(H_{1}, H_{2}\right)$ such that $A \geq 0, A^{\dagger} \geq 0$ and $R(A)$ be closed. Then $R(A)$ is a $B-C$ space iff $R\left(A^{*}\right)$ is a $B-C$ space.

Proof. We will show that if $A \geq 0, A^{\dagger} \geq 0$ and $R\left(A^{*}\right)$ is a $B-C$ space, then $R(A)$ is a $B-C$ space. This would complete the proof. For $y^{1}, y^{2} \in R(A)$ and $\phi$ a bounded linear functional on $R(A)$, consider the problem $I L P\left(y^{1}, y^{2}, \phi, I\right)$ defined on $R(A)$ :

$$
\begin{aligned}
& \text { Maximize } \phi(y) \\
& \text { subject to } y^{1} \leq y \leq y^{2}, \quad y \in R(A) .
\end{aligned}
$$

Let $x^{1}, x^{2} \in H_{1}$ such that $A x^{1}=y^{1}$ and $A x^{2}=y^{2}$. Define the sets

$$
\begin{aligned}
& S_{1}:=\left\{x \in X_{1}: A^{\dagger} A x^{1} \leq A^{\dagger} A x \leq A^{\dagger} A x^{2}\right\} \\
& S_{2}:=\left\{x \in X_{1}: A x^{1} \leq A x \leq A x^{2}\right\} .
\end{aligned}
$$

Since $A \geq 0$, and $A^{\dagger} \geq 0$, it follows that $S_{1}=S_{2}$. Setting $y=A x$, it can be seen that $\operatorname{ILP}\left(y^{1}, y^{2}, \phi, I\right)$ is the same as

$$
\begin{gathered}
\text { Maximize }\left(A^{*} \phi\right)(x) \\
\text { subject to } A x^{1} \leq A x \leq A x
\end{gathered}
$$

a problem over $H_{1}$. This is equivalent to

$$
\begin{gathered}
\text { Maximize }\left(A^{*} \phi\right)(x) \\
\text { subject to } A^{\dagger} A x^{1} \leq A^{\dagger} A x \leq A^{\dagger} A x^{2},
\end{gathered}
$$

since $S_{1}=S_{2}$.

Set $u=A^{\dagger} A x$. Then $x=\left(A^{\dagger} A\right)^{\dagger} u+z$, with $z \in N\left(A^{\dagger} A\right)=N(A)$. Also, we have $\left(A^{*} \phi\right)(x)=\phi(A(x))=\phi\left(A\left(A^{\dagger} A\right)^{\dagger} u\right)=\left(A^{*} \phi\right)\left(\left(A^{\dagger} A\right)^{\dagger} u\right)$, as $z \in N(A)$. Set $v=$ $\left(A^{\dagger} A\right)^{\dagger} u, u^{1}=A^{\dagger} A x^{1}$ and $u^{2}=A^{\dagger} A x^{2}$. Then $v, u^{1}, u^{2} \in R\left(A^{*}\right)$ and the problem above is equivalent to:

$$
\begin{gathered}
\text { Maximize }\left(A^{*} \phi\right)(v) \\
\text { subject to } u^{1} \leq u \leq u^{2},
\end{gathered}
$$


an ILP posed over $R\left(A^{*}\right)$. This has an optimal solution, as $R\left(A^{*}\right)$ is a $B-C$ space. This concludes the proof.

The following example demonstrates that the condition $A \geq 0$ (or $A^{\dagger} \geq 0$ ) is indispensable in Theorem 2.16.

EXAMPLE 2.17. Let $X_{1}=X_{2}=\mathbb{R}^{2}$ with $\mathcal{P}_{1}$ the usual cone in $\mathbb{R}^{2}$ and $\mathcal{P}_{2}$ be the lexicographic cone in $\mathbb{R}^{2}$. Then $X_{1}$ is a $B-C$ space and $X_{2}$ is not a $B-C$ space. The identity matrix is nonnegative but its inverse is not nonnegative as $\mathcal{P}_{1} \subsetneq \mathcal{P}_{2}$.

Acknowledgements. The author thanks the anonymous referee for comments and suggestions that have led to improved readability of the paper.

\section{References}

[1] A. Ben-Israel and A. Charnes, An explicit solution of a special class of linear programming problems, Operations Research 16 (1968), 1166-1175.

[2] C. W. Groetsch, Generalized Inverses of Linear Operators: Representation and Approximation, Marcel Dekker, New York, 1977.

[3] S. H. Kulkarni and K. C. Sivakumar, Applications of generalized inverses to interval linear programs in Hilbert spaces, Numer. Funct. Anal. and Optimiz. 16 (1995), 965-973.

[4] S. H. Kulkarni and K. C. Sivakumar, Explicit solutions of a special class of linear programming problems in Banach spaces, Acta Sci. Math. (Szeged) 62 (1996), 457-465.

[5] S. H. Kulkarni and K. C. Sivakumar, Explicit solutions of a special class of linear economic models, Ind. J. Pure Appl. Math. 26 (1995), 217-223.

[6] H. H. Schaefer, Banach Lattices and Positive Operators, Springer-Verlag, 1970.

[7] K. C. Sivakumar, Interval linear programs in infinite dimensional spaces, Ph.D. Dissertation, Indian Institute of Technology, Madras, 1994.

[8] K. C. Sivakumar and J. Mercy Swarna, Some results on a class of optimization spaces, Lecture Notes in Computer Science 3483, Gervasi et al. (eds.), 2005, 1341-1348.

[9] K. C. Sivakumar and J. Mercy Swarna, Linear optimization with box constraints in Banach spaces, submitted. 